\title{
Prinzipien der Gesundheitsförderung oder fünf P's sind nicht genug
}

Roland Lüthi', Werner Grüter ${ }^{2}$

In der kurzen zur Verfügung stehenden Zeit wurde innerhalb der Arbeitsgruppe "Prinzipien der Gesundheitsförderung» das Referat von Roland Lüthi diskutiert. Die 5 wichtigen «P», Präzision, Professionalität, Persönlichkeit, Popularität und Politik bildeten dabei den Rahmen. Vor allem wurde dabei das fünfte $\mathrm{P}$ «Politik» besprochen. Es wurde festgestellt, dass bei der gemeindenahen Prävention einerseits gesellschaftspolitische, anderseits aber auch wirtschafts- und parteipolitische Aspekte zu berücksichtigen sind. Gesellschaftspolitisch wirken sich besonders Massnahmen gegen Genuss- und Suchtverhalten, wie Rauchen und Alkohol, als «salonfähige Verhaltensnormen», negativ aus. Wirtschaftlich gesehen kann wirksame Prävention Interessenkonflikte auslösen, wenn beispielsweise eine (Steuern zahlende) Tabakindustrie im gleichen Kanton ihren Sitz hat, von dem aus gesundheitsfördernde Massnahmen ergriffen werden. Es braucht auch einiges an "Verhandlungsgeschick», wenn mit Ideen und Projekten eine Gemeinde angesprochen wird, deren Behördemitglieder von der «uneingeschränkten Gesundheit» der eigenen Bevölkerung überzeugt ist.

Die gesundheitsgefährdenden Ereignisse der letzten Zeit (Tschernobyl, Mühleberg, Basel) weckten bei den Diskussionsteilnehmern erneut und verstärkt den Wunsch nach äquivalenter Information. Es kann künftig nicht mehr akzeptiert werden, dass durch voreilige und unüberlegte Information die Bevölkerung irrege-

${ }^{1}$ Roland Lüthi, Dr phil. MPH, Institut für Sozial- und Präventivmedizin der Universität Bern.

${ }^{2}$ Werner Grüter, Beauftragter für Prävention, Sanitätsdepartement des Kantons Luzern. führt wird. Es sollte auch Ziel der Gesundheitsförderung sein, Behördevertreter bzw. Informationsverantwortliche so auszubilden, dass sie in Krisensituationen in der Lage sind, der Bevölkerung durch richtige Information Hilfe zu bieten.

Die 5 «P» wurden innerhalb der Arbeitsgruppe noch durch weitere ergänzt, so z. B. Partnerschaft/Zusammenarbeit. Unter diesem Begriff wurde verstanden, dass es zunehmend wichtiger wird, dass sowohl die politische Behörde wie Privatpersonen bzw. private und öffentliche Organisationen gemeinsam die Ziele der Gesundheitsförderung verfolgen. Unter dem Titel partnerschaftliche Zusammenarbeit wurde angeregt, regionale GELIKO, welche politische Öffentlichkeitsarbeit leisten, einzuführen.

Als siebtes «P» wurde der Programm- und Informationsaustausch besprochen. Einmal mehr wurde die Schaffung einer Informationsstelle postuliert, deren Aufgabe es wäre, Überblick über alle angebotenen Programme, Dienstleistungen und Projekte verschiedenster Anbieter und Partner zu schaffen. Eine solche Informationssammelstelle müsste allen Präventionsinteressierten offenstehen. Funktionieren kann sie jedoch nur, wenn jedermann bereit ist, eigene Ideen und Projekte vorzustellen.

Die Liste der präventiven «P» liesse sich ohne weiteres noch ergänzen. Als weiteres Schlagwort wurde auch Pluralismus genannt. Schlussendlich wurde auch bemerkt, dass es keine wirkungsvolle Prävention gibt, wenn die «Pegeisterung» fehlt. 